\title{
Clinical pathway for acute exacerbations of chronic obstructive pulmonary disease: method development and five years of experience
}

This article was published in the following Dove Press journal:

International Journal of COPD

28 June 2011

Number of times this article has been viewed

\section{Koichi Nishimura' \\ Maya Yasui ${ }^{2}$ \\ Takashi Nishimura ${ }^{2}$ \\ Toru Oga ${ }^{3}$}

'Department of Internal Medicine, Kaikoukai Jousai Hospital, Aichi, Japan; ${ }^{2}$ Kyoto-Katsura Hospital, Kyoto, Japan; ${ }^{3}$ Department of Respiratory Care and Sleep Control Medicine, Graduate School of Medicine, Kyoto University, Kyoto, Japan
Correspondence: Koichi Nishimura Department of Internal Medicine, Kaikoukai Jousai Hospital, 4-I Kitahata, Nakamura-ku, Nagoya 453-08I5, Japan $\mathrm{Tel}+8 \mathrm{I} 524853777$

Fax +8I 524853773

Email koichi-nishimura@nifty.com
Background: Randomized controlled trials, evidence-based medicine, clinical guidelines, and total quality management are some of the approaches used to render science-based health care services. The clinical pathway for hospitalized patients suffering from acute exacerbations of chronic obstructive pulmonary disease (AECOPD) is poorly established, although a clinical pathway is an integral part of total quality management.

Aim: To evaluate the outcomes of patients hospitalized with AECOPD in Japan, treated with a clinical pathway following published guidelines.

Methods: Prospective data were collected for patients with AECOPD admitted to a general hospital over a 5-year period since 2003. The clinical pathway was designed to establish general rules for the entire treatment protocol. The clinical pathway indicates which treatments and interventions should be performed, and when. In this study, health care providers were required to check the clinical pathway sheets to determine the next step of treatment.

Results: This study analyzed 276 hospitalizations in 165 patients. The clinical pathway was interrupted and defined as a dropout in 45 cases (16.3\%). Nine patients died during hospitalization (3.3\%). Oxygen was administered in 232 hospitalizations ( $84.1 \%$ ). Noninvasive positive pressure ventilation (NPPV) treatment was administered in 110 hospitalizations (39.9\%). The rate of intubation in those cases where NPPV treatment had been administered was $8.2 \%$ ( 9 cases out of 110). The average length of stay (LOS) was 20.3 days, and the median value was 15 days. The LOS was longer than 30 days in 34 admissions (12.3\%), mainly due to complications.

Conclusion: AECOPD can be managed using a clinical pathway. This clinical pathway could fill the gap between guidelines and clinical practice.

Keywords: COPD, AECOPD, mortality, pulmonary rehabilitation

\section{Background}

Acute exacerbation of chronic obstructive pulmonary disease (AECOPD) is one of the most common causes of acute hospital admission. These hospital admissions come with a high mortality rate and an extended impairment of the health status. Similar to diseases like pneumonia, acute myocardial infarction, and heart failure, AECOPD is common, has high morbidity, and is costly to society. However, unlike these other diseases, the underlying medical conditions responsible for AECOPD are generally unknown. Clinical guidelines have been published and updated to standardize the management of AECOPD, with the aim of improving health care and safely reducing costs. ${ }^{1}$ However, the UK National COPD Audit 2003 reported a wide variation in both the length of stay (LOS) and mortality among hospitals in the UK. ${ }^{2}$ The contributing factors remain unclear, although it appears that some of this variation can be attributed 
to patient factors. In 2006, Lindenauer and colleagues reported that only two-thirds of patients with AECOPD received the entire set of recommended care protocols in US hospitals, ${ }^{3}$ and that numerous participants received tests or treatments that were considered not beneficial. Mularski and colleagues also concluded in 2006 that AECOPD patients in the US received only $60.4 \%$ of the recommended care. ${ }^{4}$

Randomized controlled trials, evidence-based medicine, clinical guidelines, and total quality management are considered to be important methods to provide more sciencebased health care services. Integrated care pathways or clinical pathways detail tasks, sequences, timescales, and disciplines, and contain a checklist of all necessary actions. ${ }^{5}$ It was reported in 1998 that AECOPD should be included in the table of integrated care pathways in use in Britain. ${ }^{5}$ In other words, clinical pathways may be guidelines or management rules within individual hospitals, and are part of a total quality management approach to care. They aim to facilitate the introduction of clinical guidelines as well as systematic, continuing audits into clinical practice; thus, clinical pathways can provide a link between establishing clinical guidelines and practicing them. A clinical pathway is generally recommended in all common clinical settings, especially for scheduled admissions such as elective surgery.

Few reports exist on the use of clinical pathways in the management of AECOPD. The lack of established clinical pathways for AECOPD patients may be due to the difficulty associated with setting up a clinical pathway for acute or severe illnesses and critical care medicine, and to concerns about treatment failure. In addition, the number of patients hospitalized with AECOPD is still relatively low in Japan, although the prevalence of COPD in Japan has been reported to be similar to that of Western countries, based on general population studies. ${ }^{6}$ This low incidence might be another reason for the lack of an organized effort to establish a clinical pathway for AECOPD in Japan.

We hypothesized that AECOPD could be treated using a clinical pathway, and that it could fill the gap between guidelines and clinical practice. In the present study, we developed a clinical pathway and applied it to treat patients hospitalized with AECOPD in a general hospital in Japan. We then analyzed the results of 5 years of prospective observations on AECOPD patients treated with this clinical pathway.

\section{Materials and methods Development of the clinical pathway}

The present study was conducted at the Respiratory Division of Kyoto-Katsura Hospital, a general hospital that provides health care mainly for the western part of the city of Kyoto. The clinical pathway was developed in November 2002, and was implemented for the care of patients hospitalized with AECOPD. The clinical pathway was formalized by a multidisciplinary study group made up of pulmonary specialists, staff nurses, physiotherapists, pharmacists, emergency staff, and dieticians. The clinical pathway indicates which treatments and interventions should be performed, and when. In this study, the health care providers were required to check the clinical pathway sheets to determine the next step of treatment. The clinical pathway was approved by the clinical pathway committee responsible for the medical governance of the hospital.

The implemented clinical pathway for AECOPD consists of the following interventions: (1) frequent evaluations and laboratory testing, (2) pharmacological treatment, (3) instructions on the method of drug administration by a ward pharmacist, (4) respiratory management, (5) pulmonary rehabilitation during the acute phase, (6) nutritional support, and (7) early discharge planning. The core therapeutic interventions included in the clinical pathway are summarized in Table 1. In addition, the plan for antibiotics administration was adjusted based on the results of blood tests for inflammatory markers, a sputum test, and signs of pneumonia at 4 and 8 days after beginning the treatment. Intravenous infusions of aminophylline and the administration of expectorants were not included in the clinical pathway. As a general rule, when the patients were under respiratory management, such as oxygen administration and noninvasive positive pressure ventilation (NPPV) treatment, arterial blood gas analysis was performed every morning and at 30-120 minutes after changing the oxygen dose or NPPV setting. To respond to acute exacerbations of the patient's condition, the nurses were instructed to change the applied concentration of oxygen as follows: if a saturation of peripheral oxygen $\left(\mathrm{SpO}_{2}\right)<88 \%$ continues for over 30 minutes, then increase the fraction of inspired oxygen $\left(\mathrm{FiO}_{2}\right)$ one step at a time using the Ventimask $^{\circledR}$; if a $\mathrm{SpO}_{2}>93 \%$ continues over 30 minutes, then decrease the $\mathrm{FiO}_{2}$ one step at a time using the Ventimask. The initial setting for NPPV was an inspiratory positive airway pressure (IPAP) of $8 \mathrm{~cm} \mathrm{H}_{2} \mathrm{O}$, an expiratory positive airway pressure (EPAP) of $4 \mathrm{~cm} \mathrm{H}_{2} \mathrm{O}$, a backup respiratory rate of 12 breaths per minute, and oxygen supplementation to maintain the oxygen concentration before NPPV therapy. Hypercapnia was managed by an increase in the IPAP from 2 to $4 \mathrm{~cm} \mathrm{H}_{2} \mathrm{O}$ after every arterial blood gas analysis. The final IPAP was 14-20 $\mathrm{cm} \mathrm{H}_{2} \mathrm{O}$ in most hypercapnic subjects. The nurses were responsible for changing the oxygen dose 
Table I Therapeutic interventions included in the clinical pathway for the management of hospitalized patients with acute exacerbation of chronic obstructive pulmonary disease

I. Frequency of the evaluations and testing:

- Blood tests, chest x-rays, and ABG analyses on the first, fourth, eighth, and 13th day of the exacerbation

- $A B G$ analysis every morning if necessary

- Pulmonary function tests after inhalation of $200 \mu \mathrm{g}$ salbutamol using an MDI with a spacer on the day after completion of the oral glucocorticosteroid course, or before discharge

2. Pharmacological treatment:

- High-dose, frequent inhalation of a bronchodilator under supervision (introduce (1) and change to (2) in a few days)

(1) Repeated inhalation of $0.5 \mathrm{~mL}$ salbutamol using a nebulizer every hour

(2) Repeated inhalation of four puffs of salbutamol $(400 \mu \mathrm{g})+$ four puffs of oxitropium bromide $(400 \mu \mathrm{g})$ using an MDI and a spacer every 2 hours

- Oral administration of $0.5 \mathrm{mg} / \mathrm{kg}$ of prednisolone every morning for 10-14 days

- Antibiotics administration until the inflammatory markers disappear (cefazolin or piperacillin I- $2 \mathrm{~g} \times 2 \mathrm{div}$ )

- Starting inhaled corticosteroids as maintenance therapy after the completion of systemic corticosteroid administration

3. Respiratory management:

- Oxygen administration at the lowest concentration possible to maintain a $\mathrm{PaO}_{2} \geq 60 \mathrm{~mm} \mathrm{Hg}$ (start with a Ventimask ${ }^{\circledR}$ )

- Start NPPV therapy if $\mathrm{PaCO}_{2} \geq 45(-50) \mathrm{mm} \mathrm{Hg}$ or if $\mathrm{pH}$ drops to below 7.35, regardless of the oxygen concentration

4. Pulmonary rehabilitation during the acute phase:

- Visit by a physical therapist and rehabilitation at an early stage

5. Instructions on the methods of administration by a ward pharmacist

- In the case of breathing difficulties, a single dose of salbutamol by nebulizer, or $400 \mu \mathrm{g}$ salbutamol and $400 \mu \mathrm{g}$ oxitropium bromide by an MDI

- When changing to therapy using an MDI and a spacer to maintenance therapy including inhaled corticosteroids

- Inhalation techniques to be supervised by nurses following a standard checklist

6. Planning for an early discharge

7. Provision of a high-calorie diet and nutrition counseling when necessary

Abbreviations: $A B G$, arterial blood gas; MDI, metered-dose inhaler; NPPV noninvasive positive pressure ventilation; $\mathrm{PaCO}_{2}$, pressure of carbon dioxide in arterial blood; $\mathrm{PaO}_{2}$, pressure of oxygen in arterial blood.

during NPPV therapy as follows: if an $\mathrm{SpO}_{2}<88 \%$ continues over 30 minutes, then increase the $\mathrm{FiO}_{2}$ by $5 \%$ or increase the oxygen supplementation by $0.5 \mathrm{~L} / \mathrm{min}$; if a $\mathrm{SpO}_{2}>93 \%$ continues over 30 minutes, then decrease the $\mathrm{FiO}_{2}$ by $5 \%$ or lower the oxygen supplementation by $0.5 \mathrm{~L} / \mathrm{min}$.

The use of pulmonary rehabilitation during the acute phase of AECOPD is still controversial. Evidence is accumulating for the effectiveness of pulmonary rehabilitation during the recovery phase of AECOPD. ${ }^{7}$ However, the optimal time to start pulmonary rehabilitation has not been clearly established. In the present clinical pathway, a physiotherapist performed the pulmonary rehabilitation as early as possible after the vital signs became stable. The Barthel index was recorded prior to pulmonary rehabilitation, ${ }^{8}$ and the 6-minute walking test was performed as much as possible before and after the pulmonary rehabilitation. Although there is no evidence for nutritional interventions to manage AECOPD, a high-calorie diet was provided for all subjects, with nutritional counseling as necessary. Early discharge was carefully planned in all cases. At the time of discharge, the patients were further educated by instructional videos.

\section{Subjects}

This study examined those patients who were hospitalized during the 51/2-year period between January 2003 and June 2008, and who were treated according to the clinical pathway for AECOPD. The inclusion criteria for this clinical pathway were: (1) a clinical diagnosis of COPD, (2) a history of smoking (10 pack-years or greater), (3) a forced expiratory volume in one second to forced vital capacity ratio $\left(\mathrm{FEV}_{1} / \mathrm{FVC}\right)<0.7$ on or before the first day of the clinical pathway, (4) the absence of previous inflammatory changes on chest radiographs that influenced pulmonary function (for example, previous thoracoplasty or tubercular sequelae), and (5) the presence of aggravated symptoms of COPD compatible with exacerbations. Since the confirmation of airflow limitation as defined by a $\mathrm{FEV}_{1} / \mathrm{FVC}<0.7$ was necessary for inclusion, the results of previous spirometric tests were obtained whenever possible. Spirometry was required for admission even if the condition was severe, unless previous compatible spirometric results were available before admission.

The exclusion criteria were: (1) intubation on the first day of disease, (2) tracheotomy prior to the first day of disease, (3) exacerbation due to pneumothorax, and (4) exacerbation due to cardiac failure alone. When continuous treatment with a clinical pathway became impossible due to progression of the disease or complications, the case was defined as a dropout (variance).

It is well known that COPD patients suffer from the complication of community-acquired pneumonia at a high frequency. It is believed that therapeutic management of AECOPD with no clinical signs of pneumonia should be the same as that for an exacerbation of COPD as a result of pneumonia. ${ }^{9}$ In addition, it would be under-treatment if those patients with exacerbated COPD due to pneumonia were treated only for their pneumonia. Therefore, in the current clinical pathway, we decided to widen the selection criteria in this regard, and included not only AECOPD patients due to respiratory infection, but also patients with COPD complicated by pneumonia. 
In addition, we retrospectively examined as many hospital records as possible during the study period, and collected information on COPD patients who were not included in the clinical pathway.

\section{Results}

The present clinical pathway was applied to a total of 300 hospitalizations for 189 patients. Although we screened the patients based on the exclusion criteria before the clinical pathway was initiated, we found 11 cases where the clinical pathway for AECOPD was inappropriately applied. The most frequent reason for an error in the initial screening was that the patient was a never-smoker, and the attending physician applied the clinical pathway without confirming the smoking history. The second reason was that the spirometric results were not available, and that the airflow limitation was not confirmed before the initiation of the clinical pathway. These cases were excluded from our analysis.

In addition, 13 subjects were excluded from further analysis due to a disappearance of their airflow limitation. These patients successfully passed the initial screening, and an airflow limitation was confirmed at the initiation of the clinical pathway. Nevertheless, the spirometry performed before discharge revealed that the $\mathrm{FEV}_{1} / \mathrm{FVC}$ was over 0.7 (ranging from $70.6 \%$ to $94.5 \%$ ). These patients were diagnosed with a reversible airflow limitation whose initial presentation was similar to AECOPD. We believe that these cases could not be excluded initially due to the difficulty in distinguishing an asthma exacerbation from an AECOPD in some patients.

Thus, the remaining 276 hospitalizations of 165 patients were analyzed in the present study. The results are summarized in Table 2. The average age of the patients, calculated with the total number of hospitalizations as the denominator, was 74.6 years. In 97 cases (35.1\%), the patients were taken to the hospital by ambulance, and in 83 cases (30.0\%), since the chest radiographs revealed infiltration compatible with pneumonia, they were diagnosed with a pneumonic exacerbation of COPD.

Spirometry was performed in 248 out of 276 episodes after completing the treatment, and disease severity of COPD was unknown in 28 hospitalizations (10.1\%). According to the GOLD (Global Initiative for Chronic Obstructive Lung Disease) criteria, ${ }^{1}$ there were 29 (10.5\%), 81 (29.3\%), 89 (32.2\%), and 49 (17.8\%) episodes, respectively, of stage I, II, III, and IV. The post-bronchodilator $\mathrm{FEV}_{1}$ was $0.99 \mathrm{~L}$ on average, and the $\mathrm{FEV}_{1} / \mathrm{FVC}$ was $44.4 \%$. The average LOS was 20.3 days, and the median value was 15 days. The length of stay was longer than 30 days in 34 admissions $(12.3 \%)$, mainly due to complications.

Table 2 Characteristics of a total of 276 episodes from 165 patients treated by the clinical pathway for acute exacerbations of chronic obstructive pulmonary disease

\begin{tabular}{|c|c|c|c|c|}
\hline & & Number & $\%$ & \\
\hline Gender & (Male) & 234 & 84.8 & \\
\hline Smoking condition & (Current) & 51 & 18.5 & \\
\hline Pneumonia as a complication & (Present) & 83 & 30.1 & \\
\hline \multirow[t]{2}{*}{ Ambulance transportation } & (Yes) & 97 & 35.1 & \\
\hline & & Mean & Median & SD \\
\hline Age & (years) & 74.6 & 75.0 & 8.5 \\
\hline History of smoking & (pack-years) & 71 & 59 & 40 \\
\hline Length of stay & (days) & 20.3 & 15.0 & 18.9 \\
\hline \multicolumn{5}{|c|}{ Arterial blood gas before beginning treatment with variable concentration of oxygen ${ }^{a}$} \\
\hline $\mathrm{PaO}_{2}$ & $(\mathrm{~mm} \mathrm{Hg})$ & 64.5 & 58.5 & 29.4 \\
\hline $\mathrm{PaCO}_{2}$ & $(\mathrm{~mm} \mathrm{Hg})$ & 47.4 & 40.4 & 18.4 \\
\hline $\mathrm{Ph}$ & & 7.397 & 7.419 & 0.082 \\
\hline \multicolumn{5}{|c|}{ Pulmonary function tests after treatment ${ }^{b}$} \\
\hline $\mathrm{FEV}_{1}$ & $(\mathrm{~L})$ & 0.99 & 0.87 & 0.46 \\
\hline $\mathrm{FEV}_{1}$ & (\% predicted) & 51.0 & 45.9 & 23.8 \\
\hline $\mathrm{FEV}_{1} / \mathrm{FVC}$ & (\%) & 44.4 & 41.3 & 14.3 \\
\hline FVC & (L) & 2.24 & 2.15 & 0.73 \\
\hline FVC & (\% predicted) & 75.6 & 76.7 & 21.2 \\
\hline Barthel index ${ }^{c}$ & (score, $0-100)$ & 60.1 & 65.0 & 31.3 \\
\hline 6MWD before rehabilitation ${ }^{d}$ & (meter) & 199 & 175 & 143 \\
\hline 6MWD after rehabilitation ${ }^{e}$ & (meter) & 268 & 270 & 140 \\
\hline
\end{tabular}

Notes: ${ }^{a} n=275 ;{ }^{b} n=248 ;{ }^{c} n=245 ;{ }^{d} n=210 ;{ }^{e} n=210$.

Abbreviations: 6MWD, 6-minute walk distance; $\mathrm{FEV}_{1}$, forced expiratory volume in one second; FVC, forced vital capacity; PaCO ${ }_{2}$, pressure of carbon dioxide in arterial blood; $\mathrm{PaO}_{2}$, pressure of oxygen in arterial blood; $\mathrm{SD}$, standard deviation. 
The clinical pathway was completed in 231 out of 276 hospitalizations (83.7\%), but was interrupted and regarded as a dropout in 45 hospitalizations (16.3\%), see Table 3. Nine patients (3.3\%) died during hospitalization due to the following reasons: two cases each of pneumothorax-related deaths, septic shock, and cerebral infarction; one case each of nosocomial pneumonia, unconsciousness of unknown cause, and sudden death of unknown cause. One of the two pneumothorax-related deaths occurred in a subject that had a bilateral pneumothorax that developed just after the introduction of NPPV; he received subsequent mechanical ventilation with intubation, but the treatment was unsuccessful. In the second pneumothorax-related death, an autopsy revealed a collapse of the left lung in a patient who died unexpectedly on the third day after hospitalization. All of the fatal cases were due to complications after the initiation of the treatment, and were difficult to predict. Seven patients $(2.5 \%)$ were transferred to other long-term care facilities; four had families who refused their discharge to home, and the remaining three were transferred due to complications (intertrochanteric hip fracture, cerebral infarction, and interstitial pneumonia).

In 29 out of 45 dropouts, the patients did not complete the clinical pathway but were discharged home from the hospital after receiving various subsequent therapies. Among the 29 dropouts who were discharged from the hospital, in nine hospitalizations, the reason for the dropout was only because the pulmonary rehabilitation was not performed, even though all other procedures scheduled in the clinical pathway were completed. The other reasons for the dropouts were complications that appeared during the clinical pathway, including pneumonia in five cases, pleural effusion in three cases, a pneumothorax in two cases. Nine cases that required intubation and the use of a mechanical ventilator following unsuccessful NPPV treatment were also included in the dropouts.

Oxygen was administered to 232 hospitalizations with a pressure of oxygen in arterial blood $\left(\mathrm{PaO}_{2}\right)<$ $60 \mathrm{~mm} \mathrm{Hg}$ (84.1\%). NPPV treatment was administered in 110 hospitalizations with a pressure of carbon dioxide in arterial blood $\left(\mathrm{PaCO}_{2}\right) \geq 45(-50) \mathrm{mm} \mathrm{Hg}$ or a $\mathrm{pH}<7.35$ (39.9\%). Forty-four hospitalizations without respiratory failure (15.9\%) were treated without oxygen administration and without NPPV treatment, and 122 hospitalizations with a $\mathrm{PaO}_{2}<60 \mathrm{~mm} \mathrm{Hg}$ but without a $\mathrm{PaCO}_{2} \geq 45(-50) \mathrm{mm} \mathrm{Hg}$ or a pH $<7.35$ (44.2\%) were treated with oxygen administration but without NPPV treatment. There were only three dropouts (6.8\%) among these 44 hospitalizations without oxygen administration and without NPPV treatment. There were 13 cases $(10.7 \%)$ of dropouts among the 122 hospitalizations with oxygen administration but without NPPV treatment. On the other hand, there were 29 dropouts (26.4\%) out of 110 hospitalizations with oxygen administration and NPPV treatment. Therefore, the clinical pathway completion rates were $93.2 \%, 89.3 \%$, and $73.6 \%$, respectively, for these

Table 3 Outcomes of COPD-related hospitalizations during the study period

\begin{tabular}{|c|c|c|c|}
\hline \multirow[t]{11}{*}{$n=300$} & \multicolumn{3}{|c|}{ The clinical pathway applied } \\
\hline & $\mathrm{n}=1 \mathrm{I}$ & \multicolumn{2}{|c|}{ Entry criteria violation } \\
\hline & $\mathrm{n}=13$ & \multicolumn{2}{|c|}{ Disappearance of airflow limitation after discharge } \\
\hline & $\mathrm{n}=45$ & \multicolumn{2}{|l|}{ Dropout } \\
\hline & & $n=9$ & Died \\
\hline & & $\mathrm{n}=7$ & Transferred to other long-term care facilities \\
\hline & & $\mathrm{n}=29$ & Discharged to home from the hospital alive \\
\hline & $\mathrm{n}=23 \mathrm{I}$ & \multicolumn{2}{|c|}{ Treatment with the clinical pathway completed } \\
\hline & & $\mathrm{n}=44$ & Without oxygen, without NPPV \\
\hline & & $n=122$ & With oxygen, without NPPV \\
\hline & & $\mathrm{n}=110$ & With oxygen, with NPPV \\
\hline \multirow[t]{8}{*}{$n=62$} & \multicolumn{3}{|c|}{ COPD-related hospitalizations outside the clinical pathway } \\
\hline & $\mathrm{n}=25$ & \multicolumn{2}{|c|}{ AECOPD outside the clinical pathway } \\
\hline & & $\mathrm{n}=9$ & Intubations on the first day of exacerbation \\
\hline & & $\mathrm{n}=\mathrm{I}$ & Tracheotomy prior to the first day of exacerbation \\
\hline & & $\mathrm{n}=3$ & Exacerbations due to pneumothorax \\
\hline & & $\mathrm{n}=2$ & Exacerbations due to cardiac failure alone \\
\hline & & $\mathrm{n}=10$ & Prompt discharge because of very mild exacerbations \\
\hline & $\mathrm{n}=37$ & \multicolumn{2}{|c|}{ Other reasons } \\
\hline
\end{tabular}

Abbreviations: AECOPD, acute exacerbation of chronic obstructive pulmonary disease; COPD, chronic obstructive pulmonary disease; NPPV, noninvasive positive pressure ventilation. 
hospitalization groups. The rate of intubation in those cases where NPPV treatment had been administered once was $8.2 \%$ (9 cases out of 110 ).

Pulmonary rehabilitation was administered in 262 cases, and started on average 3.5 days (median 3 days) after admission. The Barthel index was recorded in 245 episodes, and the mean \pm standard deviation index value was $60.1 \pm 31.3$, ranging from 0 to 100 . The six-minute walking distance was $199 \pm 533$ and $268 \pm 660$ meters in 210 episodes before versus after pulmonary rehabilitation, respectively.

We examined the hospital records and found a total of 62 COPD-related hospitalizations that were not treated using the clinical pathway. Among these 62 episodes, we could identify the reason why the clinical pathway was not applied in 25 AECOPD episodes. In the remaining 37 episodes, it was not clear from the medical records whether the COPD was stable or not, and it was difficult to identify the reason why the clinical pathway was not applied, even though these cases appeared to match the criteria for AECOPD.

\section{Discussion}

Our analysis of AECOPD patients treated by a newly developed clinical pathway revealed two important findings. First, AECOPD can be effectively treated using a clinical pathway. Since all of the participants received all of the interventions listed in the clinical pathway, this pathway proved to be well tolerated and accepted, even in critically ill patients with AECOPD. The fact that we could apply all of the listed interventions to all of the patients suggests that the quality of care provided in our hospital had improved compared with the level of care before the introduction of the clinical pathway. Second, the rate of dropouts from the clinical pathway was relatively low (approximately 16\%), and most of the dropouts were hypercapnic cases that required NPPV treatment. This low dropout rate suggests that the clinical pathway was highly adaptable to all patient conditions. The mortality rate during hospitalization was less than $5 \%$ in the present study, further supporting the contention that the clinical pathway was safe and did not impose any significantly adverse effects, even on severely ill patients. Unfortunately, it was impossible to compare the present study with a control group treated without the clinical pathway, because the present study was not a randomized controlled trial.

AECOPD represents an extremely severe event that can occur at any point during the long course of COPD. AECOPD can be lethal, and can impair a patient's health-related quality of life severely and permanently. Thus, the first important step is to take measures to prevent AECOPD from occurring among patients with stable COPD. A comprehensive approach, including the use of drugs known to reduce the frequency of AECOPD episodes, is necessary. ${ }^{10}$ As the second step, if AECOPD does occur, then it is important to promptly change the therapeutic regimen, and, if necessary, facilitate the patient's access to medical services. Patient education and self-management involving action plans and behavior modification are among the critical issues associated with the second step. ${ }^{11}$ Studies designed to include these approaches as a part of integrated care have resulted in favorable outcomes. ${ }^{12,13}$ The third step for managing AECOPD is the actual treatment itself. For mild symptoms of AECOPD, patients can be treated by unscheduled or emergency visits. Patients suffering from moderate-to-severe symptoms of AECOPD need to be hospitalized, and our study was designed to improve the quality of treatment at this final stage of the disease.

Some clinicians may feel an aversion to the uniform treatment menu of a clinical pathway, especially because the severity of disease symptoms varies greatly among individual patients. Indeed, it would be difficult to apply a clinical pathway for acute medical conditions that included critically ill cases. It is a reasonable concern that the uniform treatment applied by the clinical pathway might result in a failure of treatment for such diseases as AECOPD, in which patients suffer from acute and severe conditions. The same treatment under the clinical pathway may be an over-treatment for some patients with mild disease or an under-treatment for some patients with severe symptoms. Furthermore, since AECOPD patients manifest qualitatively different clinical conditions, it can be argued that the treatments should be tailored to the condition of each patient. Although these concerns are valid and important to consider, it is also important to provide a simple, practical, and easily applicable plan for those practitioners working at the forefront of medical services. We believe that our study provided evidence suggesting that a clinical pathway for AECOPD is both useful and practical.

One limitation of the present study is that it was not a randomized controlled trial which examined the impact of the clinical pathway as compared with AECOPD patients treated in other ways. In the UK National COPD Audit 2003, it was reported that the inpatient mortality rate was $7.4 \%$, and the mean LOS for discharged patients was 8.3 days (median 6 days). ${ }^{2}$ In the 2005 Scandinavian audit of COPD hospitalizations, the overall in-hospital mortality was 3.7\%, and the mean LOS was 8.6 days, although the LOS was longer than 10 days in $25 \%$ of the cases. ${ }^{14}$ In the US, Stein and colleagues examined the Nationwide Inpatient Sample 
from the years 2000-2006 using five different algorithms and concluded that the in-hospital mortality was $2.0 \%-5.1 \%$, and the median LOS was 4 days in patients hospitalized for AECOPD ${ }^{15}$ Although the inpatient mortality in the Western studies can be considered comparable to our study, the LOS in the hospital, which is less than 10 days in most Western countries, is clearly longer in Japan. The LOS for all patients admitted to the Kyoto-Katsura Hospital during the period of our study was around 17 days, and this value is about the same as the average LOS for general acute hospitalizations in Japan. Any possible additional cost may be attributed to the longer stay in Japan. The reason for this difference can be attributed to the different systems of health care delivery between the Western countries and Japan. The problem in Japan is that the number of hospitals per capita is very high, but the number of staff per hospital bed is extremely low, and thus the shortage of employees in medical facilities poses a more serious problem. ${ }^{16}$ However, in Japan, there have been no reports on the therapeutic management of AECOPD, and therefore there are no references to compare the results presented in this study.

Some interventions, such as NPPV, have been shown to be effective in selecting patients enrolled in clinical trials. However, few studies are available on the use of NPPV as a routine standard medical protocol for patients with respiratory failure outside of these controlled trials. According to the report from Schettino and colleagues, ${ }^{17}$ 87 patients with AECOPD were treated by NPPV in 2001, and the rate of intubation associated with NPPV failure was $24 \% .{ }^{17}$ In our report, NPPV treatment was administered in 97 hospitalizations (40\%), and the rate of intubation in our NPPV-treated cases was $8 \%$. This favorable rate cannot be easily compared with the results reported by Schettino and colleagues, but evidence of the effectiveness of NPPV treatment to control hypercapnic AECOPD is gradually accumulating, and appears to be infiltrating clinical practice.

Ample evidence supports the effectiveness of pulmonary rehabilitation for stable COPD, but it remains controversial whether it should be applied during the acute phase of AECOPD. Evidence is now accumulating for the effectiveness of pulmonary rehabilitation during the recovery phase of AECOPD. ${ }^{7}$ However, the optimal time to start pulmonary rehabilitation has not been clearly established. ${ }^{18-20}$ In the current clinical pathway, we implemented pulmonary rehabilitation during the acute phase, and started interventions with a physiotherapist immediately after the vital signs, such as fever, became stable. More clinical trials are needed to determine when to start pulmonary rehabilitation.
In some cases, we felt that the clinical pathway would be inappropriate due to frequent and recurrent exacerbations, and we intentionally avoided using it for the benefit of these specific patients. In addition, although our clinical pathway was indicated for AECOPD inpatients, each physician may have used different criteria to determine who should be hospitalized. The absence of a uniform criterion for hospitalization might have introduced a bias into the outcomes of the clinical pathway in the present study.

Another limitation of the present study is that our limited data precluded us from examining other indices often used in the literature, such as the re-admission rate, the rate of relapses, treatment failure, and adverse effects. These parameters could be used to monitor the yearly performance of each hospital, and to compare each hospital against national standards. However, few studies have examined the cost of developing and implementing such an index, and how it may effectively change clinical practice to improve the outcomes of each hospital. The implementation of an inpatient clinical pathway for hospitalized asthmatic children led to a decrease in the LOS and a reduction in total cost, while improving the quality of care. ${ }^{21,22}$ Similarly, a clinical pathway for AECOPD should be evaluated from various viewpoints by comparing a group treated by the clinical pathway against a control group. Such a comprehensive evaluation should provide some basis to decide how a clinical pathway should be implemented for AECOPD. Another problem is that the present study was limited by the small number of cases of AECOPD included in the present series. However, this is all the patients with AECOPD admitted to this hospital during the study period. Although it has been reported that prevalence of COPD is similar to Western countries by a general population sample study, ${ }^{6}$ health care providers still feel that AECOPD is not so frequent. Since the COPD stage before AECOPD may affect the clinical pathway, the therapeutic interventions included in the clinical pathway could have been tailored to the disease severity of stable COPD before AECOPD.

In conclusion, the rate of dropouts from the clinical pathway for AECOPD was low, and most of the dropouts were hypercapnic cases that required NPPV treatment. Although there are many differences in health care delivery between Japan and Western countries, it is also important to recognize that there are many issues remaining with regard to how clinical pathways should be applied. Nevertheless, the application of evidence-based medicine and the establishment of a standard treatment protocol based on clinical guidelines will certainly allow us to use the clinical pathway for AECOPD 
as an effective strategy. We believe that the clinical pathway for AECOPD can fill the gap between the guidelines and clinical practice.

\section{Notes}

The study was conducted at the Kyoto-Katsura Hospital, Kyoto, Japan, and partly funded by the NPO Medise in Japan.

\section{Disclosure}

The authors declare that they have no competing interests.

\section{References}

1. Rabe KF, Hurd S, Anzueto A, et al. Global strategy for the diagnosis, management, and prevention of chronic obstructive pulmonary disease: GOLD executive summary. Am J Respir Crit Care Med. 2007;176(6): 532-555.

2. Price LC, Lowe D, Hosker HS, et al. UK National COPD Audit 2003: impact of hospital resources and organisation of care on patient outcome following admission for acute COPD exacerbation. Thorax. 2006; 61(10):837-842.

3. Lindenauer PK, Pekow P, Gao S, Crawford AS, Gutierrez B, Benjamin EM. Quality of care for patients hospitalized for acute exacerbations of chronic obstructive pulmonary disease. Ann Intern Med. 2006;144(12):894-903.

4. Mularski RA, Asch SM, Shrank WH, et al. The quality of obstructive lung disease care for adults in the United States as measured by adherence to recommended processes. Chest. 2006;130(6):1844-1850.

5. Campbell H, Hotchkiss R, Bradshaw N, Porteous M. Integrated care pathways. BMJ. 1998;316(7125):133-137.

6. Fukuchi Y, Nishimura M, Ichinose M, et al. COPD in Japan: the Nippon COPD Epidemiology study. Respirology. 2004;9(4):458-465.

7. Puhan M, Scharplatz M, Troosters T, Walters EH, Steurer J. Pulmonary rehabilitation following exacerbations of chronic obstructive pulmonary disease. Cochrane Database Syst Rev. 2009(1):CD005305.

8. Mahoney FI, Barthel DW. Functional evaluation: the Barthel index. $M d$ State Med J. 1965;14:61-65.
9. Lieberman D, Lieberman D, Gelfer Y, et al. Pneumonic vs nonpneumonic acute exacerbations of COPD. Chest. 2002;122(4): 1264-1270

10. Calverley PM. Reducing the frequency and severity of exacerbations of chronic obstructive pulmonary disease. Proc Am Thorac Soc. 2004;1(2): 121-124.

11. Bourbeau J, Nault D, Dang-Tan T. Self-management and behaviour modification in COPD. Patient Educ Couns. 2004;52(3):271-277.

12. Lemmens KM, Nieboer AP, Huijsman R. A systematic review of integrated use of disease-management interventions in asthma and COPD. Respir Med. 2009;103(5):670-691.

13. Wesseling G, Vrijhoef H. Acute exacerbations of COPD: recommendations for integrated care. Expert Rev Resp Med. 2008;2(4):489-494.

14. Liaaen ED, Henriksen AH, Stenfors N. A Scandinavian audit of hospitalizations for chronic obstructive pulmonary disease. Respir Med. 2010;104(9):1304-1309.

15. Stein BD, Charbeneau JT, Lee TA, et al. Hospitalizations for acute exacerbations of chronic obstructive pulmonary disease: how you count matters. COPD. 2010;7(3):164-171.

16. Nishimura K. Lung health in Japan. Chron Respir Dis. 2006;3(2): 104-105.

17. Schettino G, Altobelli N, Kacmarek RM. Noninvasive positive-pressure ventilation in acute respiratory failure outside clinical trials: experience at the Massachusetts General Hospital. Crit Care Med. 2008;36(2): 441-447.

18. Nava S. Rehabilitation of patients admitted to a respiratory intensive care unit. Arch Phys Med Rehabil. 1998;79(7):849-854.

19. Eaton T, Young P, Fergusson W, et al. Does early pulmonary rehabilitation reduce acute health-care utilization in COPD patients admitted with an exacerbation? A randomized controlled study. Respirology. 2009;14(2):230-238.

20. Troosters T, Probst VS, Crul T, et al. Resistance training prevents deterioration in quadriceps muscle function during acute exacerbations of chronic obstructive pulmonary disease. Am J Respir Crit Care Med. 2010;181(10):1072-1077.

21. Mitchell EA, Didsbury PB, Kruithof N, et al. A randomized controlled trial of an asthma clinical pathway for children in general practice. Acta Paediatr. 2005;94(2):226-233.

22. Wazeka A, Valacer DJ, Cooper M, Caplan DW, DiMaio M. Impact of a pediatric asthma clinical pathway on hospital cost and length of stay. Pediatr Pulmonol. 2001;32(3):211-216.
International Journal of COPD

\section{Publish your work in this journal}

The International Journal of COPD is an international, peer-reviewed journal of therapeutics and pharmacology focusing on concise rapid reporting of clinical studies and reviews in COPD. Special focus is given to the pathophysiological processes underlying the disease, intervention programs, patient focused education, and self management protocols.

\section{Dovepress}

This journal is indexed on PubMed Central, MedLine and CAS. The manuscript management system is completely online and includes a very quick and fair peer-review system, which is all easy to use. Visit $\mathrm{http}: / / \mathrm{www}$.dovepress.com/testimonials.php to read real quotes from published authors. 